\title{
Menopause: Changes in the Mouth Cavity and Preventive Strategies
}

\author{
Giampietro Farronato*, Cinzia Maspero, Chiara Folegatti and Lucia Giannini
}

Fondazione Irccs Cà Granda, Ospedale Maggiore Policlinico, Postgraduate School in Orthodontics, University of Milan, Italy

\section{Abstract}

Aim: Menopause is a term used to describe the permanent cessation of the primary functions of the human ovaries. Menopause typically occurs in women in midlife and signals the end of the fertile phase of a woman's life. The transition from reproductive to non-reproductive is the result of a major reduction in female hormonal production by the ovaries. During the menopause remarkable changes happen in the mouth cavity as osteoporosis, periodontal disease, Burning Mouth Syndrome (BMS) and xerostomia.

The aim of this work is to describe the changes that occur during the menopause in the mouth cavity and the preventive protocols used in the Preventive Dental Department of the University of Milan.

Material and Methods: A systematic literature review about the menopause and its modifications in the mouth cavity have been made using the data base Medline and key words as "oral health" and "menopause" have been searched.

Discussion and Conclusions: Different changes in women oral condition can be observed during menopause. These pathological conditions are typical of the hormonal phase and they must be known by clinicians in order to make a correct intervention. Problems as osteoporosis, periodontal disease, Burning mouth syndrome and xerostomia could be prevented using specific protocols.

Keywords: Woman; Menopause; Reproductive system; Female hormones; Oral health

\section{Introduction}

The "menopause" term derives from the Greek words " $\mu$ in $\nu \mu \eta v o ́ c "$

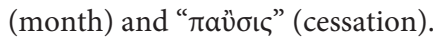

Scientifically the menopause is the physiological event that in the woman corresponds to the end of the menstrual cycle during the fertile age. In the menopause the ovarian activity finishes: the ovaries do not produce follicles and estrogens (main female hormones).

Generally speaking the term indicates only a remarkable loss of fluids, coinciding with the period of female climacteric [1].

The terms menopause and climacteric often are used wrongly as synonyms; climacteric is a period of transaction during which the function of the ovaries stops. In the women this state causes a series of changes as metabolic, sexual and psychological aspects, with a series of manifestations (symptoms) that are different from person to person. These symptoms can be more or less marked, but some of them are not connected to the menopause itself: other factors like familiar or social contest may influence them [2].

During the menopause remarkable changes happen in several districts of human body - as vascular, bone or adipose sections. They are often associated to an increased risk of insorgence of pathologies. In fact during the menopause the cardiovascular risk - as increment of the incidence of myocardial ischaemia and Myocardial infarction, ischemic stroke, artery periferic disease - is great as the risk of fractures caused by osteoporosis and disorders of the reproductive system.

The aim of this work is to describe the changes that occur during the menopause in the mouth cavity and the preventive measures used in the School of Milan.

\section{Materials and Methods}

A systematic literature review about the menopause and its modifications in the mouth cavity have been made using the data base Medline [www.ncbi.nlm.nih.gov/pubmed].
The key words have been "oral health and menopause".

The range of articles has been selected from 1980 to 2011

Only the reported articles to "Humans" have been considered using the options of the limits.

Articles published on not indexed reviews and some considerations drawn from inherent researches have been then added to. Furthermore some preventive protocols used in the School of Milan have been introduced.

\section{Results}

\section{Modifications of the mouth cavity during the menopause}

The period of the menopause is characterized by important physiological changes in the woman body, connected mainly with the reduced estrogen production. The consequences of such hormonal deficiency are marked in the cases of premature menopause, especially if corrected substitutive hormonal therapies have not been established. Some meaningful changes regard the mouth cavity as diffused pains, gums inflammation, burning, altered modulation of the four main tastes (sweet, salt, bitter and acid), dry mouth, loss of teeth and resorption of alveolar bone. The hormonal and microbiological variations influence also the mouth. A reduction of salivary secretions, dryness and irritation of the mucosae and the gums could create a gingivo-stomatitis from menopause.

*Corresponding author: Prof. Giampietro Farronato, Università di Milano, Via Commenda 10, 20100 Milano, Tel: +39 (0)55032520, E-mail: giampietro.farronato@unimi.it

Received January 09, 2012; Accepted February 01, 2012; Published February 06, 2012

Citation: Farronato G, Maspero C, Folegatti C, Giannini L (2012) Menopause: Changes in the Mouth Cavity and Preventive Strategies. J Women's Health Care 1:102. doi:10.4172/2167-0420.1000102

Copyright: (c) 2012 Farronato G, et al. This is an open-access article distributed under the terms of the Creative Commons Attribution License, which permits unrestricted use, distribution, and reproduction in any medium, provided the original author and source are credited. 
However the woman enters in a new season of the life that brings to the seniority. It increases the tendency to the osteoporosis that influences directly the resorption of the alveolar bone. From an epidemiologic point of view, there's a remarkable increasing of periodontal disease connected with age progression in particular the advanced medium form. From a therapeutic point of view, it is necessary to act with greater determination in order to eliminate not only the inflammation, but also the consequences of periodontal disease.

The main pathological oral conditions that can be manifested in menopause are:

\section{- Osteoporosis}

- Periodontal desease (Periodontitis, gingivitis, desquamative gingivitis)

- Burning mouth syndrome (BMS)

- Xerostomia

Osteoporosis: Osteoporosis is a disease characterized by a low content of calcium in bones with a resulting weakness of the skeleton and the predisposition to the fractures [3]. The relation between menopause and osteoporosis is well known: the estrogens take part in the regulation of the amount of bone calcium: the decreasing in their control, causes the reduction of the calcium level, leaving a porous and fragile structure $[4,5]$.

Even if the osteoporosis' mechanisms are not completely clear, it is believed that the estrogens promote the cellular programmed dead of the osteoclasts and therefore reduce their period of activity. The treatment with estrogens or other agents is necessary to prevent the bone loss through the block of the production of cytokines in osteoblasts and to promote osteoclasts .apoptosis Besides the deficiency of estrogens, there are other risk factors able to develop osteoporosis: in particular physical inactivity, diet with low level of calcium, constitutional thinness, long periods without menstruations, smoking, alcool, treatments with cortisonic drugs and genetical predisposition.

The teeth are based into bone structures. The deterioration, inevitable in the old persons, lead to a weakening of their anchorage. This makes easier the infiltrations in the periodontal structures that becomes more susceptible to the bacterial attack, causing gum disease $[6,7]$.

A $30 \%$ of women aged more than 60 are affected by postmenopause osteoporosis. An alteration of the equilibrium between calcium and phosphate produced by a low calcium resorption with diet and an increased calcium excretion produced by low levels of estrogens can explain some changes of bone level observed in the women during postmenopause. This involves the inferior more than the upper jaw and interests also the ossification of cartilage of the mandibularcondyloid process $[8,9]$.

Periodontitis: Periodontitis is a chronic inflammatory disease characterized by gum inflammation and alveolar bone resorption. Theperiodontal ligament located between alveolar bone and cement has an important role in maintaining the correct periodontal balance [10].

Osteoporosis is considered one of the risk factors for the periodontal disease and the loss of the teeth. Clinical observations in the women in post-menopause have confirmed an increase of the prevalence of the periodontal disease, with levels of estrogens more low, even if oral hygiene remains unchanged [11].
Gingivitis: Gingivitis is a gum inflammation, characterized by swelling, reddening, heating and bleeding derived from an increasing of plaque. This disease is reversible after removal of the responsible causes. Also some hormones can cause and increase the gingivitis. Scientific tests have demonstrated the importance of the so-called sexual hormones levels (androgens, estrogens, progestagens). There are typical gingivitis during the puberty, pregnancy and menopause: gingival inflammation can cause the insurance of a soft tissue hyperplasia called "epulide". Related with the different body modifications caused by menopause, is not surprising that $75 \%$ of the women over the 35 years accuse gingival bleeding and symptoms of gum disease. The oral symptomatology during the menopause and perimenopause includes flush or swelling up of the gums, pain, uneasiness, bleeding, dryness and change in the sense of the taste. The first step in order to prevent gingival disease and bleeding is to maintain under control the bacteria with a correct oral hygiene $[12,13]$.

Desquamative gingivitis: Desquamative gingivitis is not a single and defined disease, but it's also characterized from erythema, erosion, bubbles and desquamation of the free and supporter gum. It is characterized from red dark gingival tissue, pain and bleeding. The desquamation can be preceded from the formation of little blister. The gums are soft because the keratinocytes resistant to the food particles abrasion are absent. This condition is often present during the menopause: the consequelsomministration of progestogen estrogens can be effective. A similar gingival lesion can be due topemphigus vulgaris, Bullous pemphigoid, non lesivemucous pemphigoidor atrophic lichen plants and answers to the therapy with corticosteroids [14].

Burning mouth syndrome (BMS): Burning mouth syndrome is a typical disease of the feminine sex in particular in the menopause period. The main characteristic of the patients with BMS is the oral burning diffused in absence of other lesions mucosae and often is accompanied from other oral symptoms as xerostomia and dysgeusia. Tongue (in particular the back and the tip), palate, lips, superficial tissue in relationship with prosthesis and mouth' floor are involved. In a lot of cases burning is due to all the oral tissue. The symptomatology can be unexpected or gradual. For definition BMS remains a clinical condition in which is not possible to identify the causes certainly and therefore the aetiology remains idiopathic. In the clinical practice there are cases of oral burning in which it is possible to go back to well known causes, but even cases in which no factors can be identified. For this reasons it is useful to distinguish the idiopathic BMS from secondary BMS in which the therapeutic approach can be guided from the identification of the causes.

BMS frequently can be observed in combination with psychosomatic disorders. These patients have an expectant personality, introvert tending to the depression, to hypochondriasis and to stress. These personality disorders can be found in patients during menopause and can made worse by the perception of loosing feminine identity. Therefore the connection with the estrogenic deficit has been studied over a long time. An association between alterations of estrogenic hormones, altered function of the salivary glands with qualitative and quantitative modifications of salted and presence of oral burning has been observed in some patients. Between the causes of secondary BMS, local factors as immune system diseases, infections, xerostomia, Migratory glossitis and others can be reported. A specific and resolute treatment of BMS does not exist. Many drugs have been empirically employed in the treatment of the BMS with variable results. The local antihistaminics and anaesthetics can be used to calm the burning at least 
temporary. The administration somministrazione of drugs as placebo it sometimes Useful. In the psychosomatic forms, the patient approach will have to be of psychological type before still pharmacologic one.

Xerostomia: Xerostomia is a pathological condition with insufficient and altered salivary secretion. It is diffused particularly in the old persons, expecially women and the incidence is approximately a quarter of the general population.

Xerostomia can be permanent or temporary. The salivary production returns normal for a temporary period in the diseases with high fever and dryness or liquids loss - as diabetes or hyperthyroidism.

The more important causes of permanent xerostomia are the following ones:

- $\mathrm{x}$-rays of neck and head in the treatment of tumors.

- surgical removal of the salivary glands.

- Sjogren's syndrome (it is an autoimmune disease that causes a destruction of salivary and lacrimal glands) [15].

- xerostomia caused by drugs [16].

Mycoticnfections, dental caries, prosthetic problems and difficulty to feed itself can be associated to the xerostomia. Moreover salivary reduction involves the presence of a thick plaque and an increasing of detritus that can cause periodontal infections. The treatment of xerostomia happens is pilocarpina's administration that increases the salivary production.It can be improvedby topical application of salivary substitutes even if they do not have a large efficiency.

Saliva: The estrogens have got a regulation role of qualityand quantity of salivary secretion [17].

Salivary reduction connected with an estrogen reduction, is a factor that compromises dental prostheses adhesion, reducing the possibility to chew, to taste the food and to facilitate the digestion.

This condition essentially achieves also an alteration of phonesis and mouth confort, feeling known as "dry mouth". It is clinically defined as "xerostomia" and it is due to a reduction of salivary secretion.

The parameters considered important to know salivary imbalances in menopause are:

- Every day profile of Cortisol

- Progesterone

- Estradiol

- Estriol

Cortisol is a steroidhormone produced by adrenal glands and derived from cortisone. In particular it belongs to the category of glucocorticoid, of which ago part also corticosterone (the less assets). Because of endocrine glands' Atrophy during the aging, the majority of hormones produced in smaller amounts/in less uqantity. However the levels of cortisol approximately remain constant or decrease in lower level (approximately a 15-25\% in less regarding the juvenile values).

Progesterone is important for the good course of the pregnancy. Beyond the fifty years progesterone becames important for the woman: in fact a too much low level of progesterone increases menopause's disorders.

Estradiol is the main estrogenic hormone produced by the ovaries during the cycle and it is synthetized from the teca cells of ovary, from the testicle and in minimal part from the surrenalic cortex. Evaluation of plasmatic levels of this hormone represents an index of ovarian functionality and the axis hipothalamus-hipophiisis-gonads. Estradiol's levels are different according to the menstrual cycle: during menopause the normal value comes down to 5-27 ng/dl.

Estriol is one of several conjugated estrogens that can be used against the menopause's symptoms; It is considered a weak estrogen regarding estradiol and ethinylestradiol [18].

Different clinical tests have drawned attention to estriol as useful support during the menopause. Its effects are:

- it alleviates the symptoms of the menopause (Hot flashes and insomnia);

- it prevents the loss of bone density;

- it reduces the vaginal dryness and it promotes the health of the urinay system in the women in menopause and post menopause.

\section{Rules for Correct Nourishment}

A correct nourishment is important also in menopause. In fact it prevents the possibility of a weight increase or obesity and provides the specific necessities (as an increase of calcium or folates) [19]. Osteoporosis starting in menopause can be controlled with a correct intervention on nourishment with a good contribution of calcium and vitamin D improving the effects of drugs therapy [20]. This can limit the use of nutritional supplements in menopause [21].

\section{Preventive Protocols}

Hormonal alterations of woman body make easier problems as gum disease and tooth decay. A greater attention is necessary in oral hygiene with frequent controls 9 . Hormonal increase in the menstrual phase causes a greater reactivity of the gums regarding the plaque. During the pregnancy the $60-75 \%$ of women is subjected to pregnancy gingivitis, characterized from reddening, edema and bleeding. Also the menopause, that it is characterized by a reduced estrogen production, has different conseguences on the mouth cavity: gums inflammation, altered modulation of the four main tastes (sweet, salt, bitter and acid) and dry mouth. Prevention of periodontal infections may been explained carefully, emphasizing the reasons of a frequent Calculus buildup removal in addition to a meticulous every day hygiene. Regular cleaning visits should be made two times at least in year while in cases of bleeding, halitosis or gum inflammation, a dental visit becomes obligatory. Furthermore twice daily toothbrushing and flossingmust be daily after every meal. The substitutive hormonal therapy can be effective also to prevent oral pathologies, but it can have contra indication $[22,23]$.

In literature the term Hormone Replacement Therapy (HRT) indicates the administration of estrogens by themselves or in combination with progesterone ones in women in postmenopause, given in order to compensate the following estrogenic deficiency during to the cessation of the ovarian production. The association of progesterone is justified only in patients with uterus for endometrial protection. For the patients who use only estrogenic hormone (without uterus) this therapy is known as Estrogen Replacement Therapy (ERT). During the patient's, it's not useful to underline the specific connection between oral condition and menopause because the patient could have the tendency to overstimate this connection and to understimate the necessity of measures of individual care. Because of the importance 
of local factors, the attention goes addressed towards the necessity of regular professional interventions and towards the increase of the efforts for the everyday plaque control [24-26]. The symptoms of physical and emotional changes must be considered during the appointments' development. Probably the patient could be stiff and irritable:

- The relationship between patient and clinician should be established with courtesy and personal attention.

- It is important to give attention to the details - in particular waiting without reason and using instruments without efficiency or calm is not recommended.

- Boring patient during the conversation is not useful.

Oral health preservation is particularly important to maintain natural teeth. Teeth extraction should be limited in order to avoid prosthetic solutions $[27,28]$.

\section{Discussions and Conclusions}

During menopause and following periods, different changes in women oral condition can be observed. These pathological conditions are typical of the hormonal phase that interests all the woman body and they must be known by clinicians in order to make a correct intervention. Problems as osteoporosis, periodontal disease, Burning mouth syndrome and xerostomia could be prevented using specific protocols.

Periodontal tissue is influenced by hormonal action. A rigid prevention must be applied to minimize inflammatory problems, gingival and mucosal infection and risk of teeth loss.

Patients with removable prosthesis should be subjected to periodic hygienic control to prevent the possible lesions that could be found on soft oral tissue. Clinician may know the possible hormonal therapies and all the drugs' problems that could be present in the mouth cavity during the therapy as xerostomia.

This work presents the preventive measures used in the School of Dentistry of Milan. In particular the correct psychological approach to patients during the menopause, the development of hygienic protocols during professional appointments and the importance of every day plaque control are recommended. For these reasons other studies are necessary because of a new attention that menopause needs from a social and psychological point of view.

\section{References}

1. Baldoni M, Farronato F, Farronato GP, Salvato A (1986) [Preventive Dental Methodology]. Prev Assist Dent 12: 7-15.

2. Farronato G, Chiari A, Cristina A (1985) Esame al SEM della placca batterica in corso di terapia ortognatodontica. Atti Del Xx Congresso Nazionale Ciocmf Saint Vincent.

3. Jeffcoat MK, Geurs NC, Lewis CE (2003) Osteoporosis and periodontal bone loss. Clin Calcium 13: 577-581.

4. Bonjour JP, Ammann P, Chevalley T, Rizzoli R (2001) Protein intake and bone growth. Can J Appl Physiol S153-S166.

5. Palmer RM, Wilson RF, Hasan AS, Scott DA (2005) Mechanisms of action of environmental factors--tobacco smoking. J Clin Periodontol 6: 180-195.
6. Ullom-Minnich P (1999) Prevention of osteoporosis and fractures. Am Fam Physician 60: 194-202.

7. Hallworth RB (1998) Prevention and treatment of postmenopausal osteoporosis Pharm World Sci 20: 198-205.

8. Reinhardt RA, Payne JB, Maze CA, Patil KD, Gallagher SJ, et al. (1999) Influence of estrogen and osteopenia/osteoporosis on clinical periodontitis in postmenopausal women. J Periodontol 70: 823-828.

9. von Wowern N, Klausen B, Kollerup G (1994) Osteoporosis: a risk factor in periodontal disease. J Periodontol 65: 1134-1138.

10. Albright F, Bloomberg F, Smith PH (1994) Postmenopausal osteoporosis. Tr Assoc Am Phys 55: 298-305.

11. Loos BG, John RP, Laine ML (2005) Identification of genetic risk factors for periodontitis and possible mechanisms of action. J Clin Periodontol 6: 159-179.

12. Michalowicz BS, Aeppli D, Virag JG, Klump DG, Hinrichs JE, et al. (1991) Periodontal findings in adult twins. J Periodontol 62: 293-299.

13. Maspero C, Carletti V, Giannini L, Borsetti B (2009) Studio microbiologico e sistematico della placca batterica: significato diagnostico e prognostico. Rivista Italiana di Igiene Dentale 5: 9-16.

14. Farronato G, Chiari A, Cristina A (1985) Esame al SEM della placca batterica in corso di terapia ortognatodontica. Atti Del Xx Congresso Nazionale Ciocmf Saint Vincent.

15. Shapira L, Wilensky A, Kinane DF (2005) Effect of genetic variability on the inflammatory response to periodontal infection. J Clin Periodontol 6: 72-86.

16. Madau M, Stromhmenger L (2003) Prevenzione e promozione della salute orale in età pediatrica. Quintessenza, Milano, Italy.

17. Maspero C, Carletti V, Giannini L, Farronato D (2010) Disturbi de comportamento alimentare, ruolo dell'odontoiatra e dell'igienista dentale. II Dentista Moderno 50-55.

18. Masoni S, Panattoni E, Rolla P, Rossi M, Giuca MR, et al. (1991) [Stomatological problems related to pregnancy. A statistical study]. Minerva Stomatol 40: 791 796.

19. Seely EW, Walsh BW, Gerhard MD, Williams GH (1999) Estradiol with or without progesterone and ambulatory blood pressure in postmenopausal women. Hypertension 33: 1190-1194.

20. Maspero C, Carletti V, Giannini L, Farronato D, Zanotto S (2008) Prevenzione e igiene orale in gravidanza. Prevenzione \& Assistenza Dentale 4: 1-12.

21. Giannì E, Farronato GP, Camia R, Balestrieri L (1988) [Orthognatic surgical therapy: dietary problems. 2]. Mondo Ortod 13: 45-56.

22. Sherman B, Wallace R, Bean J, Schlabaugh L (1981) Relationship of body weight to menarcheal and menopausal age: implications for breast cancer risk. $\mathrm{J}$ Clin Endocrinol Metab 52: 488-493.

23. Farronato G, Anselmi M, Balestrieri I, Calderini A (1988) Metodologie preventodontiche. Follw-up a 3anni Oris Medicina 8: 11-21.

24. Baldoni M, Farronato F, Farronato GP, Salvato A (1986) [Preventive dental methodology]. Prev Assist Dent 12: 7-15.

25. Oakley GP Jr, Erickson JD (1995) Vitamin A and birth defects. Continuing caution is needed. N Engl J Med 333: 1414-1415.

26. Staessen JA, van der Heijden-Spek JJ, Safar ME, Den Hond E, Gasowsk $\mathrm{J}$, et al. (2001) Menopause and the characteristics of the large arteries in a population study. J Hum Hypertens 15: 511-518.

27. Montalcini T, Gorgone G, Gazzaruso C, Perticone F, Pujia A (2007) Large brachial diameter is associated with carotid atherosclerosis in postmenopausal women. Atherosclerosis.

28. Turner M, Aziz SR (2002) Management of the pregnant oral and maxillofacial surgery patient. J Oral Maxillofac Surg 60: 1479-1488. 